\title{
Glucagon receptor inactivation leads to $\alpha$-cell hyperplasia in zebrafish
}

\author{
Mingyu Li ${ }^{1}$, E Danielle Dean², Liyuan Zhao',3, Wendell E Nicholson ${ }^{2}$, Alvin C Powers ${ }^{1,2,4}$ \\ and Wenbiao Chen ${ }^{1}$ \\ ${ }^{1}$ Departments of Molecular Physiology and Biophysics, Vanderbilt University School of Medicine, Light Hall, \\ Room 711, 2215 Garland Avenue, Nashville, Tennessee 37232, USA \\ ${ }^{2}$ Division of Diabetes, Endocrinology, and Metabolism, Department of Medicine, Vanderbilt University, Nashville, \\ Tennessee 37232, USA \\ ${ }^{3}$ Third Institute of Oceanography, State Oceanic Administration, Xiamen 361005, China \\ ${ }^{4}$ Veterans Affairs Tennessee Valley Healthcare System, Nashville, Tennessee 37212, USA
}

Correspondence should be addressed to W Chen

Email

wenbiao.chen@ vanderbilt.edu

\begin{abstract}
Glucagon antagonism is a potential treatment for diabetes. One potential side effect is $\alpha$-cell hyperplasia, which has been noted in several approaches to antagonize glucagon action. To investigate the molecular mechanism of the $\alpha$-cell hyperplasia and to identify the responsible factor, we created a zebrafish model in which glucagon receptor (gcgr) signaling has been interrupted. The genetically and chemically tractable zebrafish, which provides a robust discovery platform, has two gcgr genes (gcgra and gcgrb) in its genome. Sequence, phylogenetic, and synteny analyses suggest that these are co-orthologs of the human GCGR. Similar to its mammalian counterparts, gcgra and gcgrb are mainly expressed in the liver. We inactivated the zebrafish gcgra and gcgrb using transcription activator-like effector nuclease (TALEN) first individually and then both genes, and assessed the number of $\alpha$-cells using an $\alpha$-cell reporter line, Tg(gcga:GFP). Compared to WT fish at 7 days postfertilization, there were more $\alpha$-cells in gcgra-I-, gcgrb-I-, and gcgra-I-;gcgrb-I- fish and there was an increased rate of $\alpha$-cell proliferation in the gcgra-l-;gcgrb-I- fish. Glucagon levels were higher but free glucose levels were lower in gcgra-l-, gcgrb-/-, and gcgra-I-;gcgrb-I- fish, similar to Gcgr-I- mice. These results indicate that the compensatory $\alpha$-cell hyperplasia in response to interruption of glucagon signaling is conserved in zebrafish. The robust $\alpha$-cell hyperplasia in gcgra-1-;gcgrb-/- larvae provides a platform to screen for chemical and genetic suppressors, and ultimately to identify the stimulus of $\alpha$-cell hyperplasia and its signaling mechanism.
\end{abstract}

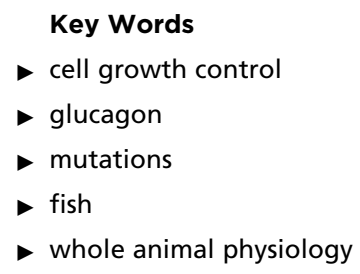

Journal of Endocrinology (2015) 227, 93-103

\section{Introduction}

Glucagon is a peptide hormone secreted by pancreatic $\alpha$-cells. It is the main counter-regulatory hormone of insulin. Glucagon acts primarily on liver through glucagon receptor (GCGR) to regulate hepatic glucose production (Mayo et al. 2003). The GCGR is a class B G-protein-coupled receptor that couples to Gs (G protein subunits) and activates adenylate cyclase to increase intracellular levels of cAMP upon ligand binding (Brubaker \& Drucker 2002). The cAMP subsequently activates protein kinase A, leading to increased glycogenolysis and gluconeogenesis and decreased glycogen synthesis (Jiang \& Zhang 2003). GCGR is primarily expressed in the liver, but is also detected in other tissues, including $\beta$-cells, heart, intestinal smooth muscle, kidney,

Published by Bioscientifica Ltd. 
brain, and adipose tissue (Hansen et al. 1995, Ali \& Drucker 2009, Kedees et al. 2009). Gcgr expression in hepatocytes is upregulated by glucose and factors known to raise intracellular cAMP levels (Abrahamsen et al. 1995).

Glucagon raises the blood glucose and excess glucagon action is seen in both types 1 and 2 diabetes (Lund et al. 2014). Knockout of Gcgr in mice results in mild hypoglycemia and decreased adiposity, and prevents diabetes induced either by streptozotocin or by a high fat diet (Gelling et al. 2003, Conarello et al. 2007, Lee et al. 2011), supporting glucagon antagonism as a potential treatment for both types of diabetes. However, as compensation to loss of glucagon action, Gcgr-/- mice also exhibit $\alpha$ - and $\delta$-cell hyperplasia, hyperglucagonemia (Gelling et al. 2003), and may develop pancreatic endocrine tumors (Gelling et al. 2003, Yu et al. 2011). Similarly, loss of GCGR function in humans is associated with hyperglucagonemia, $\alpha$-cell hyperplasia, and endocrine tumor (Zhou et al. 2009). These undesirable consequences of glucagon antagonism must be mitigated if anti-glucagon treatment is to be useful and safe. A key question about the compensatory $\alpha$-cell hyperplasia is the responsible mechanism. Liver-specific inactivation of Gcgr or its downstream transducer $\mathrm{G}_{\mathrm{s}}$ alpha subunit (Gs $\alpha$ ) in mice results in similar phenotype, suggesting the signal originates in the liver (Chen et al. 2005, Longuet et al. 2013). Furthermore, $\alpha$-cell hyperplasia occurs in islets transplanted beneath the renal capsule, indicating the responsible factor circulates (Longuet et al. 2013). However, neither the factor that stimulates $\alpha$-cell hyperplasia nor its signaling mechanism is known.

The zebrafish has emerged as a discovery platform for understanding molecular mechanisms of vertebrate biology. Its small size and transparent larvae make zebrafish an extremely useful for genetic and chemical modifier screens to discover critical components of a biological process (Lieschke \& Currie 2007). Many signaling pathways and transcription factors important for mammalian pancreatic $\alpha$ - and $\beta$-cell development are conserved in zebrafish (Biemar et al. 2001, Field et al. 2003, Hesselson et al. 2011, Maddison \& Chen 2012). Here we investigated the biology of the glucagon system in zebrafish in an effort to determine if the zebrafish might serve as a discovery platform for signals and mechanisms that regulate $\alpha$-cell mass. We found that zebrafish have two gcgr genes, gcgra and gcgrb. We functionally characterized their gene products, and found that $\alpha$-cells hyperplasia occurs in $g c g r a-$ and/or $g c g r b$-deficient zebrafish larvae.

\section{Materials and methods}

\section{Zebrafish lines and maintenance}

Zebrafish (Danio rerio) were raised in an Aquatic-Habitats system on a $14 \mathrm{~h}: 10 \mathrm{~h}$ darkness cycle at $28^{\circ} \mathrm{C}$. Embryos were obtained by natural cross and kept in embryo rearing solution and staged according to standard methods (Kimmel et al. 1995). In this study, Tg(gcga:GFP) (Zecchin et al. 2007) was used to mark $\alpha$-cells. All procedures have been approved by the Vanderbilt University Institutional Animal Care and Use Committee.

\section{Transcription activator-like effector nuclease-mediated mutagenesis of gcgra and gcgrb}

The candidate transcription activator-like effector nuclease (TALEN) target sequences of gcgra and gcgrb were identified online using TALEN Targeter (https://tale-nt. cac.cornell.edu/). The target sequences for the gcgra TALEN pair are 5'-GCCCTGCCCAACACTACAGT-3' (left) and 5'-GTATCTGCCCTGGCACAAGG-3' (right). The target sequences for the $g c g r b$ TALEN pair are 5'-CTTCTGGGAAATCTCTGAAG-3' (left) and 5'-TGGAGGATCTACACCAATG-3' (right). The TALENs were assembled using the 'Golden Gate TALEN Assembly' Kit (Cermak et al. 2011). TALEN expression vectors were linearized and used as the template for capped mRNA synthesis uing T3 mMessage mMachine Transcription Kit (Ambion, Austin, TX, USA) according to the manufacturer's instructions. Equal amounts of capped mRNAs were mixed and co-injected into one-cell stage zebrafish embryos at the dose of $200 \mathrm{pg} / \mathrm{embryo}$. T7 Endonuclease I (NEB, Ipswich, MA, USA) digestion was used to evaluate the efficiency of the TALENs. Briefly, a $\sim 400 \mathrm{bp}$ fragment was amplified from genomic DNA isolated from injectedembryos at $24 \mathrm{~h}$ postfertilization, and purified product was digested and cleaved products resolved from intact products by agarose gel electrophoresis.

A gcgra allele with $22 \mathrm{bp}$ deletion, designated as gcgra $^{\text {vu600 }}$ and a $g c g r b$ allele with 1 bp deletion, designated as $g c g r b^{\text {vu601 }}$, were selected for additional analysis. For gcgra $^{\text {vu }}{ }^{\text {voo }}$ carriers, PCR products amplified using gcgra-Mu22-F1 and gcgra-TN2-GTR following gel electrophoresis with 4\% NuSieve GTG agrose (Lonza, Rockland, ME, USA) to distinguish the WT (180 bp) and mutant $(158 \mathrm{bp})$. For $g c g r b^{\text {vu601 }}$ carrriers, PCR products amplified using gcgrb-TN2-GTF and gcgrb-TN2-GTR were digested with PstI. PCR product from carriers could not be digested.

Published by Bioscientifica Ltd. 


\section{Cloning and sequence analysis}

A putative gcgra gene was identified in Ensembl (http:// www.ensembl.org; Ensembl ID: ENSDART00000156788). A pair of primers, gcgra-Long-F1 and gcgra-Long-R1 (Supplementary Table 1, see section on supplementary data given at the end of this article), was used to amplify the entire open reading frame (ORF) from zebrafish cDNA using Q5 High-Fidelity DNA Polymerase (NEB). To obtain the ORF of $g c g r b$, the cDNA sequence was first determined using $3^{\prime}$ - and 5'-RACE based on the Ensemble ID: ENSDART00000021878. The entire ORF was subsequently cloned using primers gcgrb-Long-F1 and gcgrb-Long-R1.

Phylogenetic analysis was performed by the neighborjoining method using the MEGA4 Software (The Biodesign Institute, Tempe, AZ, USA) and full-length amino acid sequences. Bootstrap analyses were run on 1000 replicates. The genomic structure of zebrafish gcgra and gcgrb was determined by the Blat program (http://genome.ucsc.edu/ cgi-bin/hgBlat) using the cloned full-length cDNA sequence as query to search zebrafish Assembly Zv9 (July 2010). Synteny analysis was carried out based on zebrafish Assembly Zv9 (http://www.ensembl.org/Danio_rerio/ Info/Index) and human Build GRCh38 (http://www. ensembl.org/Homo_sapiens/index.html), and from zebrafish and human synteny map respectively.

\section{RNA extraction, RT-PCR, and quantitative real-time RT-PCR}

Total RNA was isolated from adult zebrafish and embryos using TRIzol reagent (Invitrogen Life Technologies) and digested by the RQ1 RNase-Free DNase (Promega) to remove any genomic DNA contamination. First strand cDNA was synthesized using M-MLV (Promega) with oligo $(\mathrm{dT})_{16}$ as primer. RT-PCR was carried out using GoTaq Flexi DNA Polymerase (Promega) at MyCycler Thermal Cycler (Bio-Rad). The primers are listed in Supplementary Table 1 . $\beta$-actin was used as an internal control. RT-PCR products were fractionated by $1.5 \%$ agarose gel electrophoresis, stained with ethidium bromide, and photographed using ChemiDoc XRS Gel Documentation System (Bio-Rad). Quantitative real-time RT-PCR (qRT-PCR) was performed in a CFX96 system (Bio-Rad). After a 3-min incubation at $95^{\circ} \mathrm{C}$, the amplification was performed as follows: $95^{\circ} \mathrm{C}, 10 \mathrm{~s} ; 60^{\circ} \mathrm{C}, 30 \mathrm{~s}$ for 40 cycles. Each assay for an unknown sample was performed in triplicate. mRNA levels were calculated using $2^{-\Delta \Delta C t}$ method (Livak \& Schmittgen 2001) and presented as relative (fold) levels normalized to the level of $\beta$-actin.

\section{CAMP accumulation}

Zebrafish glucagon, Gcga (HSEGTFSNDYSKYLETRRAQDFVQWLMNA) and Gcgb (HSEGTFSNDYSKYLETRRAQDFVQWLMNS) peptides were synthesized by GENEWIZ (Cambridge, MA, USA). Mouse glucagon was from Sigma-Aldrich (Sigma G2044). ORF of zebrafish gcgra and $g c g r b$ were subcloned in the pcDNA $3.1+$ at KpnI and EcoRI sites. Mouse Gcgr ORF was subcloned in the pcDNA $3.1+$ at EcoRI and XhoI sites. HEK293T cells are co-transfected with CRE-luciferase reporter plasmid and pcDNA3.1, pcDNA3.1-gcgra, pcDNA3.1-gcgrb, and pcDNA3.1-Gcgr using Dilute LipoD393 (SignaGen Laboratories, Gaithersburg, MD, USA). Forty-eight hours after transfection, cells were washed and dissociated, then evenly seeded into each well of a 96-well plate. Different final concentrations of glucagon peptides were added to each well with forskolin as the control. After a $4 \mathrm{~h}$ incubation at $37^{\circ} \mathrm{C}$, ONE-Glo Luciferase mixture was added to each well. After a 5 min incubation at room temperature, the signal in the plate was read using SpectraMax M5 Microplate Reader (Molecular Devices, Sunnyvale, CA, USA). Nonlinear regression analysis was performed to calculate cAMP concentrations using GraphPad Prism software (GraphPad Software, Inc., La Jolla, CA, USA).

\section{Free glucose assay}

Free glucose of zebrafish larvae was determined using the Amplex Red Glucose/Glucose Oxidase Assay Kit (Life Technologies). A pool of ten larvae was homogenized in $100 \mu$ l of sample buffer. The homogenate was cleared by centrifugation. According to the manufacturer's instructions, $10 \mu \mathrm{l}$ of supernatant (equivalent of one larva) was measured. The reaction was incubated for $30 \mathrm{~min}$ at room temperature and fluorescence was measured at $\mathrm{Ex} / \mathrm{Em}=$ $535 / 590 \mathrm{~nm}$ in a SpectraMax M5 Microplate Reader (Molecular Devices). At least three ten-fish pools of each genotype were measured.

\section{Whole mount in situ hybridization and whole mount immunofluorescence}

Digoxigenin-labeled antisense and sense RNA probes were synthesized in vitro using the linearized plasmid as a template. Hybridization was carried out as described previously (Ni et al. 2012). The larval zebrafish of $T g$ (gcga:GFP) were stained using monoclonal anti-glucagon antibody (Sigma G2654) using standard techniques as described previously (Li et al. 2014).

Published by Bioscientifica Ltd. 


\section{Proliferation analysis}

Proliferation was analyzed using the Click-iT EdU Alexa Fluor 594 Imaging Kit (C10339; Invitrogen). To identify proliferating $\alpha$-cell, embryos were incubated with $1 \mathrm{mmol} / \mathrm{l}$ 5-ethynyl-2-deoxyuridine (EdU) for $24 \mathrm{~h}$ starting at 4 days postfertilization (dpf). EdU was detected according to published protocols (Li et al. 2013). All images were collected using a Zeiss LSM710 (Carl Zeiss, Jena, Germany).

\section{Counting of $\alpha$-cells}

After fixation in $4 \%$ paraformaldehyde overnight at $4{ }^{\circ} \mathrm{C}$, larvae were washed with $1 \times$ PBS plus $0.1 \%$ Tween-20 (PBST) and flat mounted in Aqua-Mount (Richard-Allan Scientific, Kalamazoo, MI, USA) with their right side facing the coverslip. The larvae were flattened just to disrupt the islet slightly to allow better resolution of $\alpha$-cell. The $\alpha$-cells were counted according to the GFP signal using a Zeiss AxioImager under a $40 \times$ lens or using confocal projections taken by Zeiss LSM710 under a $40 \times$ lens (Carl Zeiss).

\section{Whole fish glucagon content measurement}

Glucagon content in zebrafish was measured by RIA in the Vanderbilt Hormone Assay Core similar to mouse whole pancreata measurements (Longuet et al. 2013). The two zebrafish peptides share $76 \%$ identity with mammalian glucagon. Ten zebrafish larvae (7 dpf) were collected in $0.11 \mathrm{~N} \mathrm{HCl}$ in water and homogenized with a Kontes motorized homogenizer and pestle. The samples were solubilized for $72 \mathrm{~h}$ at $4{ }^{\circ} \mathrm{C}$ while rotating. Cleared extracts were stored at $-80^{\circ} \mathrm{C}$ after centrifugation until use. We used a glucagon RIA Kit (Glucagon KGND1, Siemens Healthcare Diagnostics, Los Angeles, CA, USA) according to the manufacturer's protocol with modifications to increase the sensitivity. Specifically, the glucagon antibody was diluted twofold and the glucagon ${ }^{125} \mathrm{I}$ diluted to 5000 c.p.m./tube. The antibody detected the two zebrafish peptides with equal affinity, albeit with a small reduction (right shift) compared to the mammalian peptide (data not shown). There was no difference in the slope of the binding curve between mammalian, zebrafish a, or zebrafish b glucagon $(P>0.05)$.

\section{Statistical analysis}

Data are means and s.e.m. Data were analyzed by one-way ANOVA followed by Newman-Keuls post hoc test or $t$-test (SPSS). Significance was accepted at $P<0.05$.

\section{Results}

\section{Cloning and characterization of two gcgr genes from zebrafish}

Two putative $g c g r$ genes were found in Ensembl zebrafish genome assembly (http://www.ensembl.org/Danio_rerio/ Info/Index). Subsequently, primers were designed to amplify both ORFs. The ORF of gcgra and gcgrb were 1563 and $1527 \mathrm{bp}$ and encoding proteins of 520 and 508 amino acids respectively. Structural analysis revealed that both zebrafish gcgra and gcgrb had a structure similar to their mammalian counterparts, which contained an extracellular domain, seven transmembrane domains, and an intracellular domain.

We next generated a phylogenetic tree using the neighbor-joining method. As shown in Fig. 1A, zebrafish Gcgr forms a cluster with known GCGRs with a high bootstrap support value and well separated from GIP receptors, GLP1 receptors and GLP2 receptors. Synteny analysis revealed that zebrafish gcgra is located on chromosome 3, gcgrb is located on chromosome 1 , while the human GCGR locus is on chromosome 17. To explore the possible synteny relationship between zebrafish $g c g r$ and human $G C G R$, we compared genes surrounding the $G C G R$ loci. Several neighboring genes of $G C G R$, namely BAIAP2, AATK, SLC25A10, FN3KRP, TBCD, ZNF750, $B 3 G N T L 1$, and METRNL, also had their orthologs near zebrafish gcgra or $g c g r b$ (Fig. 1B). This conserved synteny relationship provides further evidence that gcgra and gcgrb are both orthologous to human GCGR.

\section{Expression patterns of gcgra and gcgrb mRNA}

RT-PCR detected $g c g r a$ and $g c g r b$ mRNA in all stages examined, including 1, 2, 3, 5, $7 \mathrm{dpf}$, and 3 weeks of age (Fig. 2A). In adult tissues, gcgra mRNA was most abundant in liver, followed by brain, muscle, and heart, but was undetectable in intestines. Similarly, gcgrb mRNA was most abundant in liver, but was also expressed in intestines at a level similar to brain and was undetectable in muscle and heart. Therefore, gcgra and gcgrb have distinctive expression in muscle, heart, and intestines (Fig. 2B).

\section{Functional characterization of zebrafish Gcgra and Gcgrb}

There are two glucagon genes in zebrafish, $g c g a$ and $g c g b$ (Argenton et al. 1999, Cruz et al. 2010). We synthesized Gcga and Gcgb peptides and determined their dose-response curve in HEK293T cells expressing Gcgra,

Published by Bioscientifica Ltd 
A

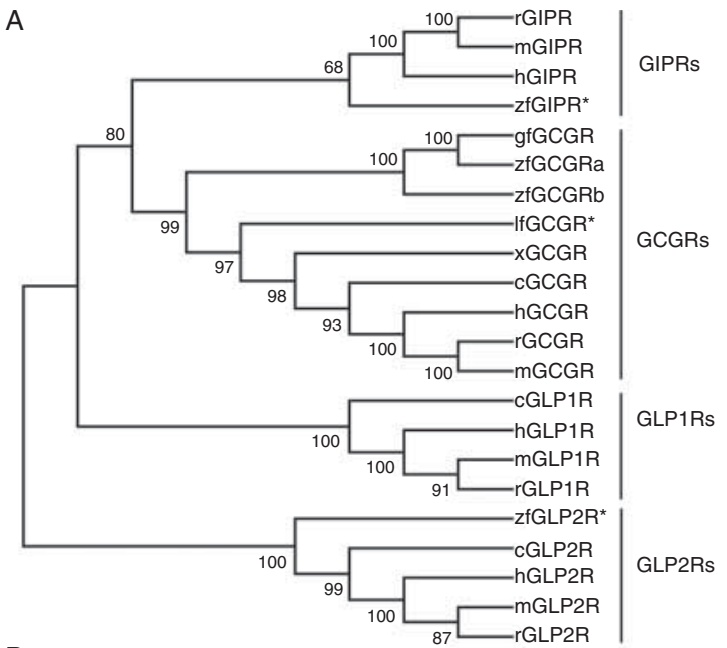

B

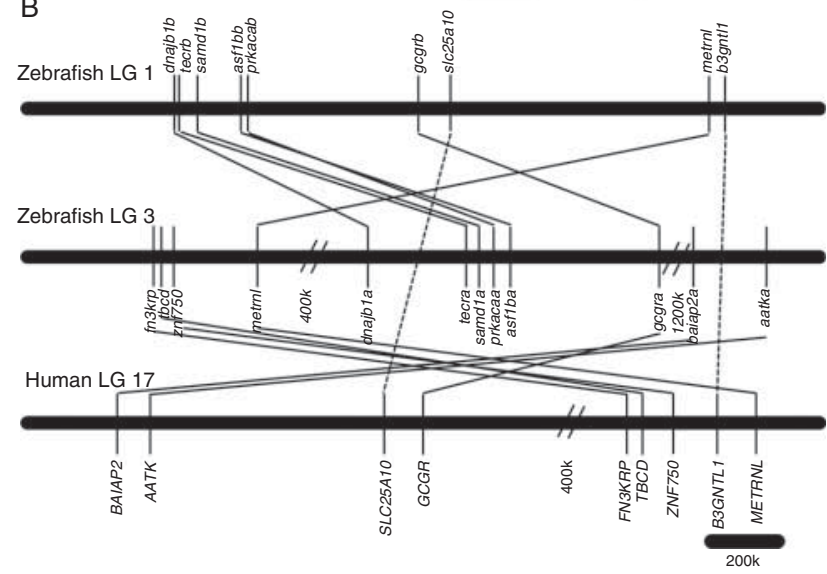

Figure 1

Identification of zebrafish gcgra and gcgrb. (A) Phylogenetic analysis of glucagon receptors. Full-length amino acid sequences of various receptors for glucagon-like peptides were analyzed using the neighbor-joining method. Posterior probability values (1000 runs) for branches of the tree are given on nodes. GIPR, glucose-dependent insulinotropic polypeptide receptor; GLP1R, glucagon-like peptide 1 receptor; GLP2R, glucagon-like peptide 2 receptor. The prefixes used are: $h$, human; $m$, mouse; $r$, rat; $c$, chicken; x, Xenopus laevis; zf, zebrafish; gf, goldfish; If, lungfish. *Predicted sequences. (B) Conserved synteny of gcgra and gcgrb to human GCGR. Zebrafish gcgra and gcgrb are located on chromosome 3 and chromosome 1 respectively. They share several syntenic genes of human GCGR on chromosome 17.

Gcgrb, and mouse GCGR using the CRE-luc reporter system. As shown in Fig. 2, zebrafish Gcga and Gcgb stimulated cAMP accumulation in cells expressing zebrafish Gcgra with EC50 value of 4.16 and $14.6 \mathrm{nM}$ respectively. Mouse GCG had a much higher $(1.96 \mu \mathrm{M})$ EC50 on zebrafish Gcgra (Fig. 2C and Table 1). The mouse and two zebrafish glucagon peptides activated zebrafish Gcgrb at similar EC50 values (Gcga, $27.95 \mathrm{nM}$; Gcgb, $14.88 \mathrm{nM}$; and GCG, $40.46 \mathrm{nM}$; Fig. 2D and Table 1). However, Gcga and Gcgb only weakly activated mouse
GCGR, contrasting to an EC50 value of $8.33 \mathrm{nM}$ for mouse GCG. These data suggested that the two zebrafish GCGRs have similar characteristics in responding to zebrafish glucagons (Gcga and Gcgb), but only Gcgrb responds to mouse glucagon. Zebrafish glucagons have a limited activity on the murine GCGR.

\section{Generation of gcgra and gcgrb mutations using TALEN}

To investigate the physiological function of Gcgra and Gcgrb, we generated loss-of-function alleles of gcgra and gcgrb using TALEN-mediated mutagenesis. The TALENs targeted the third exon of $g c g r a$ and second exon of $g c g r b$ respectively. From the TALEN mRNA-injected founders, we obtained one germline mutation in gcgra with a $22 \mathrm{bp}$ deletion $\left(g c g r a^{\text {vu }}{ }^{\circ 00}\right)$, and one germline mutation in $g c g r b$ with 1 bp deletion $\left(g c g r b^{\text {vu601 }}\right)$, both resulting in readingframe shift and premature stop codon (Fig. 3A, B, C, D, E, $\mathrm{F}, \mathrm{G}$ and $\mathrm{H}$ ). QRT-PCR analysis showed that gcgra mRNA levels were not changed in the $g c g r b^{\text {vu }} 601 / g c g r b^{\text {vu601 }}$ mutants, but were decreased in gcgra $^{\text {vu60o }} / g_{c g r a}$ vu600 $(0.65 \pm 0.02)$ and $g c g r a^{v u 600} / g c g r a^{\text {vu60o }} ; g c g r b^{v u 601} / g c g r b^{\text {vu601 }}$ $(0.55 \pm 0.02)$. The levels of $g c g r b$ mRNA also were not changed in the $\operatorname{gcgra}^{\text {vu60o }} /$ gcgra ${ }^{\text {vu600 }}$ mutants, but were decreased in $g c g r b^{\text {vu601 }} / g c g r b^{\text {vu601 }}$ mutants $(0.67 \pm 0.05)$ and gcgra $^{\text {vu600 }} /$ gcgra $^{\text {vu600 }} ;$ gcgrb $^{\text {vu601 }} / g c g r b^{\text {vu601 }}$ mutants $(0.56 \pm$ 0.16) (Fig. 3I). The results suggest that there is no compensatory regulation of the two glucagon genes. The reduction of the mutant mRNA levels is likely due to nonsense-mediated decay of mRNA.

\section{Disruption of zebrafish gcgra and gcgrb causes pancreatic $\alpha$-cell hyperplasia in larvae}

To determine the role of GCGRs in the regulation of pancreatic $\alpha$-cell mass, we crossed the gcgra ${ }^{\text {vuroo }}$ and gcgrb ${ }^{\text {vu601 }}$ into $T g(g c g a: G F P)$ fish whose $\alpha$-cells are labeled with GFP (Zecchin et al. 2007), allowing cell number determination. We confirmed by immunofluorescence that all GFP positive cells in the islet of the transgenic fish express glucagon (Supplementary Fig. 1). We then determined the number of $\alpha$-cells in WT and mutants at 7 dpf. Compared to $T g(g c g a: G F P)$ with WT $g c g r$ genes $(20.63 \pm 0.53$; mean \pm s.E.M.), there was an increase of $\alpha$-cell number in the principal islet of gcgra $^{\text {vu60o }}$ / gcgra $^{\text {vu60o }} ; \operatorname{Tg}(g c g a: G F P) \quad(27.73 \pm 1.50 ; \quad P<0.01) \quad$ and $g c g r b^{\text {vu601 }} / g c g r b^{\text {vu601 }} ; \operatorname{Tg}(g c g a: G F P) \quad(23.64 \pm 0.54 ; P<0.01)$ (Fig. 4A and B). There, increase was even greater in gcgra $^{\text {vu600 }} /$ gcgra $^{\text {vu600 }} ; g_{\text {cgrb }}$ vu601 $/ g c g r b^{\text {vu601 }} ; \mathrm{Tg}($ gcga:GFP) fish $(32.89 \pm 0.84$; $P<0.001$; Fig. $4 \mathrm{~A}$ and $\mathrm{B})$. To determine

Published by Bioscientifica Ltd. 

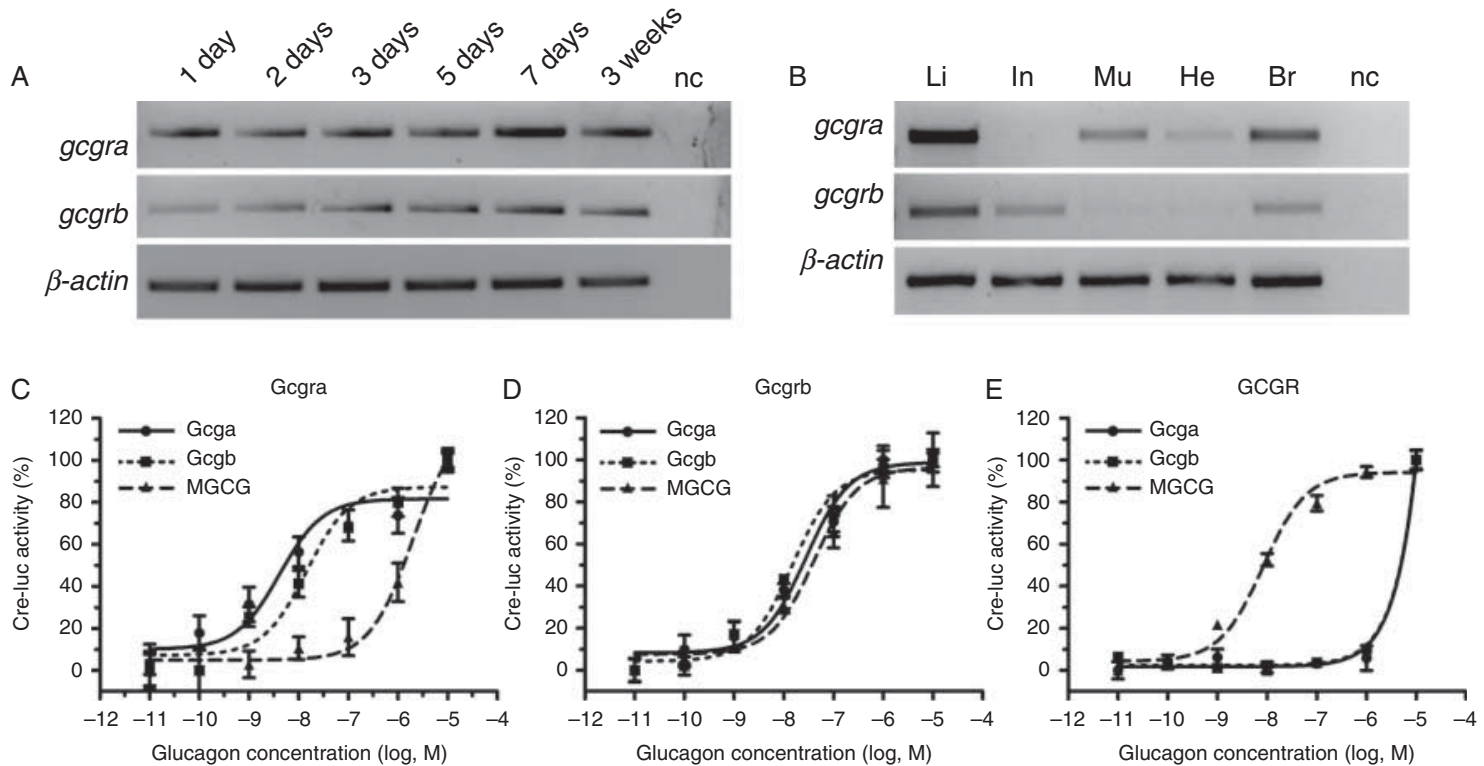

\section{Figure 2}

Expression patterns of gcgra and gcgrb and pharmacological profiles of their gene products. (A) RT-PCR analysis gcgra and gcgrb mRNA at different stages of development. The upper panel is gcgra mRNA, the middle panel is gcgrb mRNA, and lower panel is $\beta$-actin mRNA. The developmental stages are shown at the top as days or weeks post fertilization. nc, negative control. (B) RT-PCR analysis gcgra and gcgrb mRNA in different tissues in adult fish. Total RNA was isolated from liver (Li), intestine (In), muscle (Mu), heart $(\mathrm{He})$, and brain $(\mathrm{Br})$ from adult zebrafish. $\mathrm{nc}$, negative control.

when the difference occurs, we followed $\alpha$-cell number from 3 to $8 \mathrm{dpf}$ in $\mathrm{Tg}(\mathrm{gcga}: \mathrm{GFP})$ and $\mathrm{gcgra}^{\text {vu } 600} / \mathrm{gcgra}^{\text {vu60o }}$; $g c g r b^{\text {vu601 }} / g c g r b^{\text {vu601 }} ; \operatorname{Tg}(g c g a: G F P)$ fish. The increase of $\alpha$-cell number was observed as early as $4 \mathrm{dpf}(19.38 \pm 0.65$ vs $25.00 \pm 0.96, P<0.001$; Fig. 4 C). The largest single-day increase of $\alpha$-cells occurred from 4 to $5 \mathrm{dpf}$ in gcgra $^{\text {vu60o/ }}$ $g_{c g r a}{ }^{\text {vu60o }} ; g c g r b^{\text {vu601 }} / g c g r b^{\text {vu601 }} ; \mathrm{Tg}(g c g a: G F P)$ fish (Fig. 4C). These data suggest that disruption of zebrafish gcgra and gcgrb causes supernumerary $\alpha$-cells in zebrafish.

To determine whether $\alpha$-cell replication contributed to the supernumerary $\alpha$-cells in zebrafish $g c g r$ mutants, we incubated $4 \mathrm{dpf}$ larvae with EdU for $24 \mathrm{~h}$ to label replicating cells. Compared to $T g(g c g a: G F P)(1.71 \pm 0.56)$, there were a significant increase of EdU positive $\alpha$-cells
( $C, D$ and E) Dose-response curves of zebrafish and mouse glucagon receptors (GCGRs) to zebrafish and mouse glucagon peptides as measured by CAMP-dependent CRE-luciferase expression. HEK293T cells were co-transfected with pCRE-luc and expression vector pCDNA3.1(+) containing zebrafish Gcgra (C), zebrafish Gcgrb (D), and mouse GCGR (E). The cells were exposed to different concentrations of zebrafish glucagon-a (Gcga), glucagon-b (Gcgb), and mouse glucagon (mGCG) peptides $48 \mathrm{~h}$ after transient transfection. Each data point is the mean of triplicates.

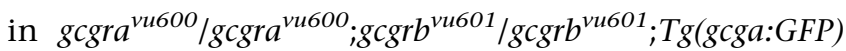
$(4.56+0.99 ; P<0.05$; Fig. 5). The number of EdU positive $\alpha$-cells trended to be increased in both $g_{c g r a}{ }^{\text {vu60o/ }}$ gcgra $^{\text {vu60o }} ; \mathrm{Tg}(\mathrm{gcga}: G F P)(3.11 \pm 0.45)$ and gcgrb $^{\text {vu601/ }}$ $g c g r b^{v u 610} ; \operatorname{Tg}(g c g a: G F P)(2.75 \pm 0.45)$ fish, although the difference was not statistically significant (Fig. 5). These data suggest that the supernumerary $\alpha$-cells are partially due to replication.

\section{Mild hypoglycemia and hyperglucagonemia in gcgr-deficient zebrafish}

To assess whether supernumerary $\alpha$-cells in $g c g r$ deficient zebrafish contributes to hyperglucagonemia,

Table 1 EC50 of glucagons to glucagon receptors (GCGRs) as measured by CAMP-mediated luciferase activity. EC50 values represent glucagon concentrations that elicit $50 \%$ of the maximum response to GCGRs. EC50 (upper panel in each cell) and $95 \%$ Cls (lower panel in each cell) were shown. Each data point was the average of triplicates

\begin{tabular}{llc}
\multicolumn{1}{c}{ Receptor } & & Gcga \\
\cline { 1 - 1 } Gcgra & & $4.161 \times 10^{-9}\left(1.125 \times 10^{-9}-1.539 \times 10^{-8}\right)$ \\
Gcgrb & & $2.798 \times 10^{-8}\left(1.354 \times 10^{-8}-5.779 \times 10^{-8}\right)$ \\
mGCGR & & 0.03148 \\
& &
\end{tabular}

http://joe.endocrinology-journals.org DOI: $10.1530 / \mathrm{JOE}-15-0284$
(C) 2015 Society for Endocrinology Printed in Great Britain

\begin{tabular}{c} 
Gcgb \\
\hline $1.461 \times 10^{-8}\left(5.827 \times 10^{-9}-3.664 \times 10^{-8}\right)$ \\
$1.488 \times 10^{-8}\left(8.770 \times 10^{-9}-2.524 \times 10^{-8}\right)$ \\
$\sim 0.02404$
\end{tabular}

\section{mGCG}

$1.962 \times 10^{-6}\left(8.530 \times 10^{-7}-4.513 \times 10^{-6}\right)$

$4.046 \times 10^{-8}\left(1.562 \times 10^{-8}-1.048 \times 10^{-7}\right)$

$8.329 \times 10^{-9}\left(5.481 \times 10^{-9}-1.266 \times 10^{-8}\right)$ 


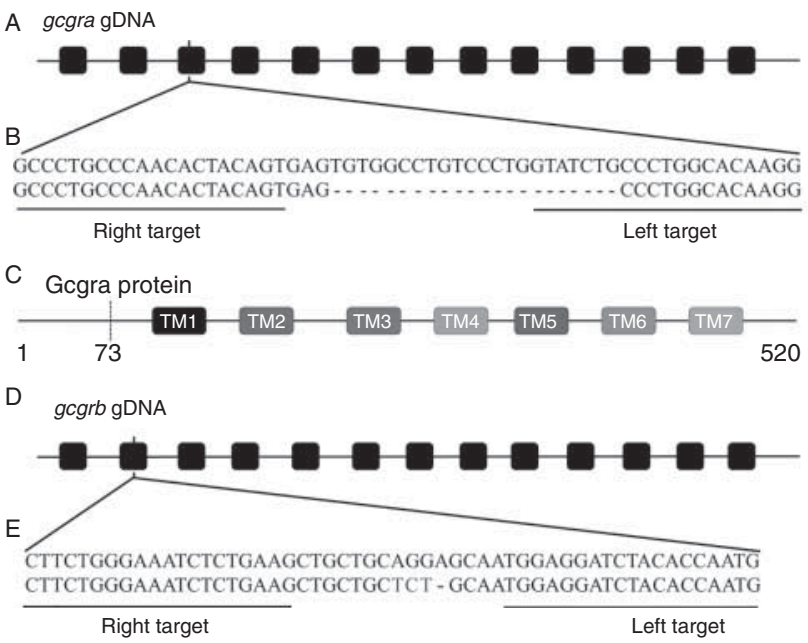

Figure 3

Generation of $g_{c g r a} V U 600$ and $g c g r b^{V U 601}$. (A, B, C, D, E and F) Schematic representations of TALEN targets and mutant alleles. Both gcgra (A) and gcgrb (D) consists of 13 exons (filled box). The TALEN pairs target exons 3 and 2 respectively. The sequences of the target regions are aligned to the selected alleles for gcgra (B) and gcgrb (E). The location of the truncation in the mutant gene product for gcgra (C) or gcgrb (F) are indicated with a

we determined the mRNA and protein levels of glucagon at $7 \mathrm{dpf}$. Compared to WT controls, the levels of $g c g a$ and $g c g b$ mRNA were both significantly increased in gcgra $^{\text {vu600 }} /$ gcgra $^{\text {vu600 }}$ and gcgra $^{\text {vu600 }} /$ gcgra $^{\text {vu600 }} ;$ gcgrb $^{\text {vu601 }} /$ $g c g r b^{\text {vu601 }}$ larvae, but not in $g c g r b^{\text {vu601 }} / g^{c g r b}{ }^{\text {vu601 }}$ larvae (Fig. 6A and B). No change in insulin expression was detected (Fig. 6C). Expression analysis by in situ hybridization confirmed an increase of $g c g a$ and $g c g b$ in the islet of the double mutants (Supplementary Fig. 2). The analysis also indicated elevated intestinal expression of the preproglucagon mRNA in the double mutants (Supplementary Fig. 2), suggesting increased Glp1 in the zebrafish mutants as in GCGR-/- mice (Gelling et al. 2003). To determine whether the increase of gaca and $g c g b$ mRNA in the islet resulted in more glucagon peptide, we measured the total glucagon content using RIA. Compared to total glucagon in the control group $(3.23 \pm 0.30 \mathrm{pg} / \mathrm{fish}), \quad g c g r a^{\text {vu } 600} / g c g r a^{\text {vu } 600}(4.07 \pm 0.16 \mathrm{pg}$,

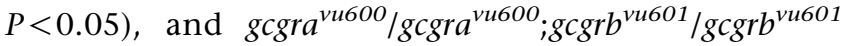
$(5.83 \pm 0.35 \mathrm{pg}, P<0.001)$ had more glucagon per fish (Fig. 6D). The increased glucagon content was consistent with an increased $\alpha$-cell number in the mutants.

To assess the effect of GCGR deficiency on gluconeogenesis, we measured free glucose levels at $7 \mathrm{dpf}$. Compared to WT control, there was a significant decrease in gcgra $^{\text {vu600 }} / g^{c g r a} a^{\text {vu600 }}, g^{c g r b}{ }^{\text {vu601 }} / g c g r b^{\text {vu601 }}$ and

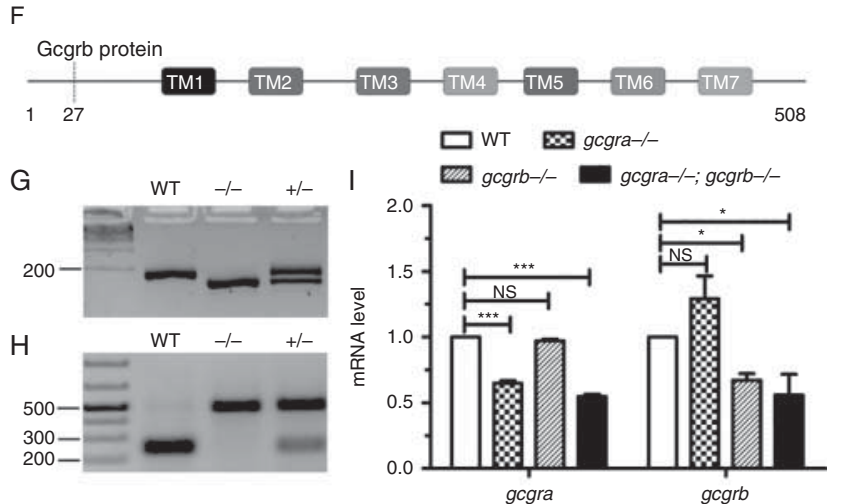

dotted line. (G and H) Genotyping of WT, heterozygous, and homozygous of gcgra (G) and gcgrb (H). (I) Real-time PCR analysis of gcgra and gcgrb mRNA levels. The results were from three independent experiments. All the values shown are means \pm s.E.M., ${ }^{*} P<0.05$ and $* * * P<0.001$ by one-way ANOVA.

$g_{c g r a}{ }^{v 4600} / g c g r a^{v u 600} ; g c g r b^{v u 601} / g c g r b^{v u 601}$ mutants (Fig. 5E). To determine whether this is due to changes in the expression of gluconeogenic genes, we measured the expression of phosphoenolpyruvate carboxykinase (soluble) (pck1), pck2 (mitochondrial), glucose-6-phosphatase (g6pca.1) mRNA by qRT-PCR (Fig. 6F, G and H). Compared to WT control, there was a significant reduction of $p c k 1$, pck2, and g6pca.1 mRNA levels in $g c g r a^{v u 600 /}$ $g_{c g r a}{ }^{v u 600} ; g_{c g r b}{ }^{v u 601} / g_{c g r b}{ }^{v u 601}$ larvae (Fig. 6F, G and H). However, no significant change in pck1 and g6pca.1 expression was found in $g c g r a^{v u 600} / g c g r a^{v u 600}$ fish.

\section{Discussion}

Antagonism of glucagon action is a potential therapeutic approach in type 2 diabetes. To advance glucagon antagonism as a therapy requires a thorough understanding of its compensatory responses to $\alpha$-cell hyperplasia. However, the molecular explanation for the $\alpha$-cell hyperplasia is unknown. In an effort to identify the responsible factor or factors, we have used the genetic tractability of zebrafish to determine if the underlying compensatory mechanism is conserved. In this study, we characterize the GCGRs of zebrafish and demonstrate that $\alpha$-cell hyperplasia occurs in zebrafish, like in rodents and humans, when GCGR signaling is interrupted.

Published by Bioscientifica Ltd. 

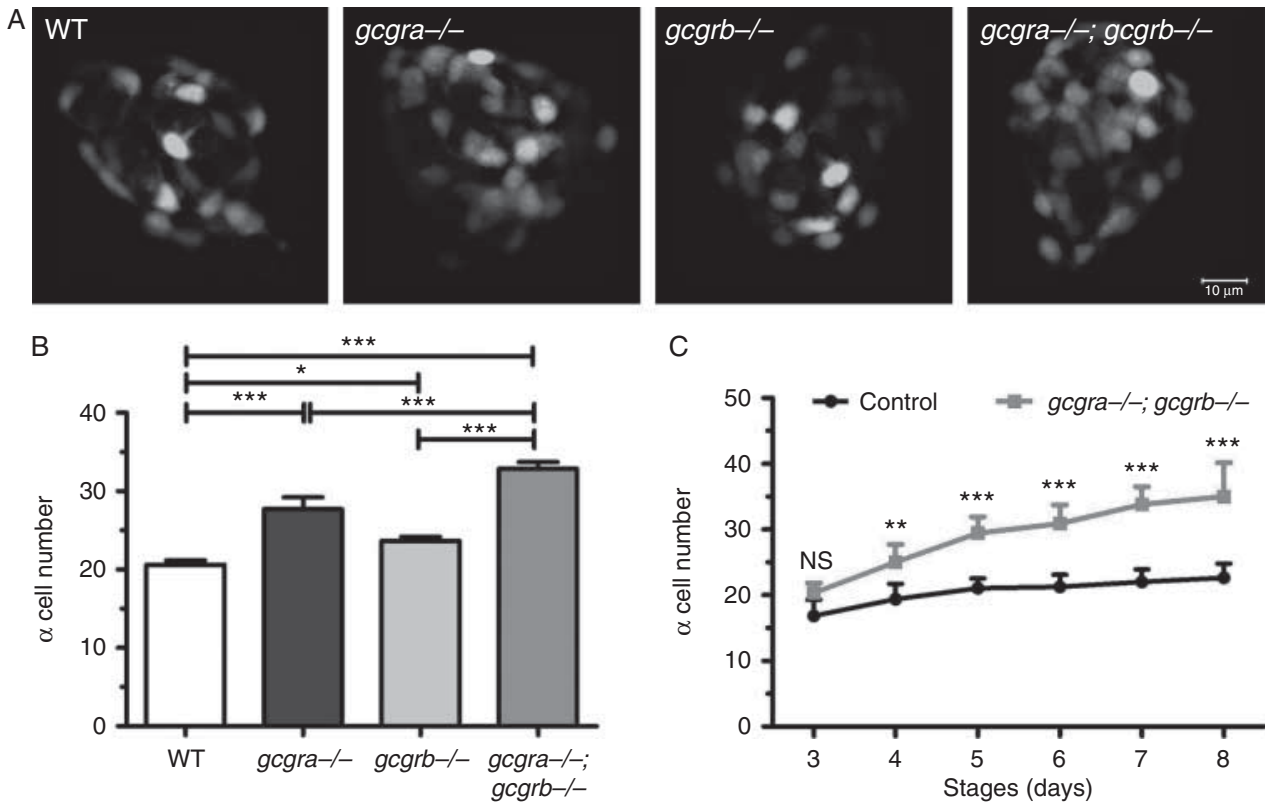

Figure 4

Homozygous mutants of gcgr genes develop $\alpha$-cell hyperplasia. (A) Representative images of the principal islet of $T g$ (gcga:GFP), gcgra-I-; Tg(gcga:GFP), gcgrb-1-;Tg(gcga:GFP), gcgra-1-;gcgrb-1-; $T g$ (gcga:GFP) at $7 \mathrm{dpf}$. The images are confocal projections; scale bars indicate $10 \mu \mathrm{m}$. (B) Quantification of the $\alpha$-cell number in different

We show that the zebrafish have two $g c g r$ genes and that both are functional. This is likely due to the additional round of genome duplication in the teleost lineage (Venkatesh 2003). As with many other gene duplications, gcgra and $g c g r b$ are likely preserved because of functional partitioning. While both are expressions in the liver and brain, gcgra is not expressed in the intestines whereas $g c g r b$ is not expressed in the heart and muscle (Fig. 2B). Nevertheless, their protein products

genotypes of zebrafish at $7 \mathrm{dpf}, n=8-12 . * P<0.05$ and $* * * P<0.001$ by one-way ANOVA. (C) $\alpha$-cell number in Tg(gcga:GFP), and gcgra-l-; gcgrb-I-;Tg(gcga:GFP) from 3 to $8 \mathrm{dpf}, n=8-16$. The values shown are means \pm s.E.M., $* * P<0.01$ and $* * * P<0.001$ by $t$-test.

respond similarly to both zebrafish glucagon peptides, Gcga and Gcgb, and couple to Gs to increase intracellular cAMP. An interesting pharmacological difference between the two receptors is that Gcgra is much less responsive to mammalian glucagon than Gcgrb. A surprising finding is that both zebrafish glucagon peptides fail to activate mouse GCGR (Fig. 2E). This is similar to goldfish glucagon, which fails to bind rat GCGR (Chow et al. 2004).

A

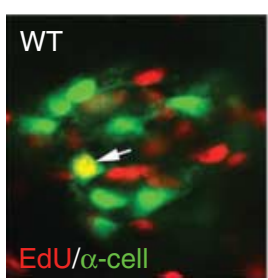

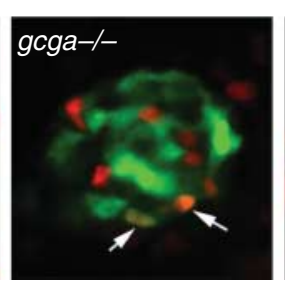

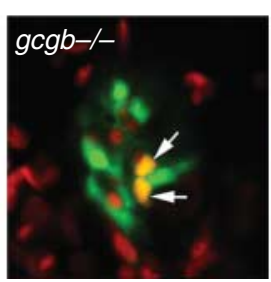

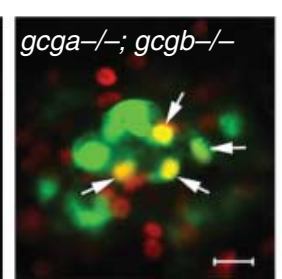

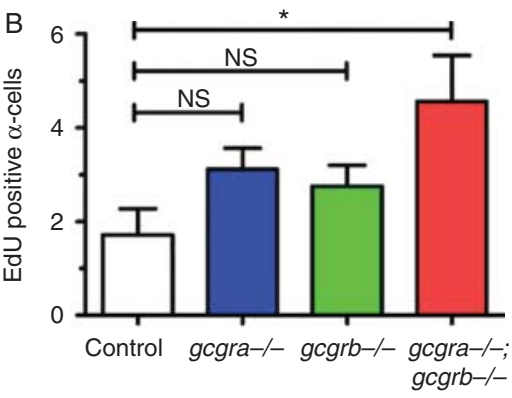

Figure 5

Increased $\alpha$-cell proliferation in gcgr-deficient zebrafish larvae. (A) Representative images of different genotypes larvae by EdU staining. Arrows indicated the EdU (red) positive $\alpha$-cell (green). The images are confocal projections; scale bar indicates $10 \mu \mathrm{m}$. (B) Quantification of EdU labeled $\alpha$-cells, $n=7-10$. All the values shown are means \pm s.E.M., ${ }^{\star} P<0.05$ by one-way ANOVA.

Published by Bioscientifica Ltd 

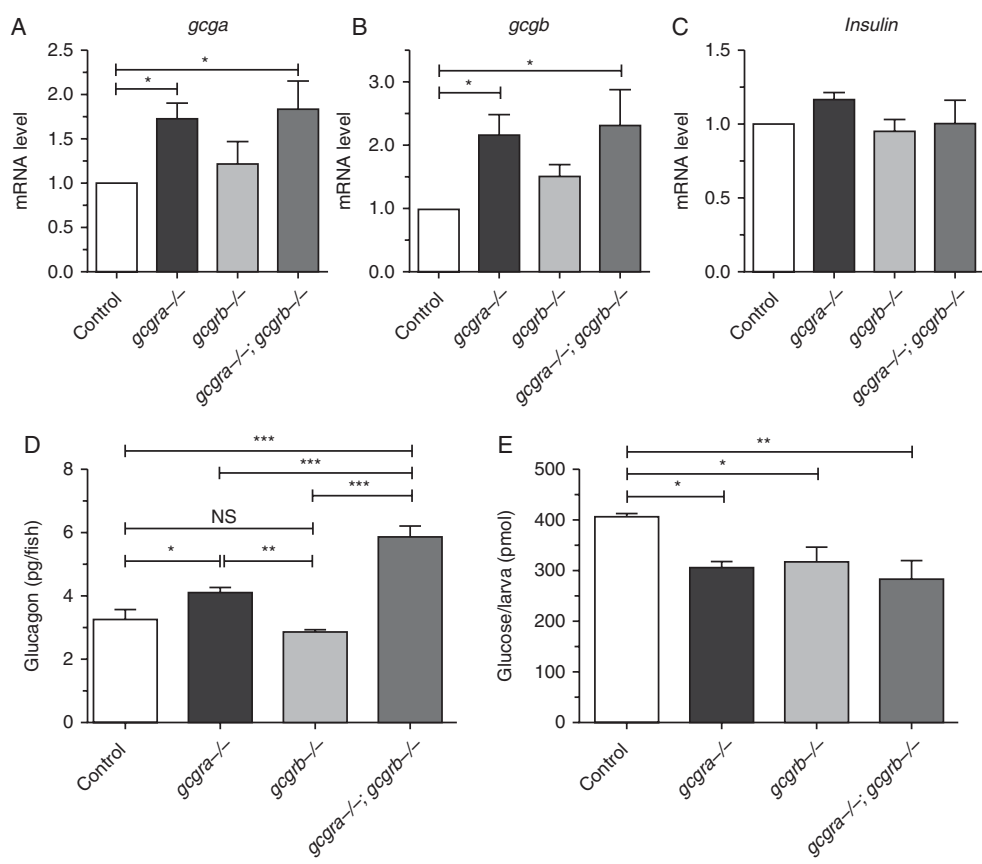
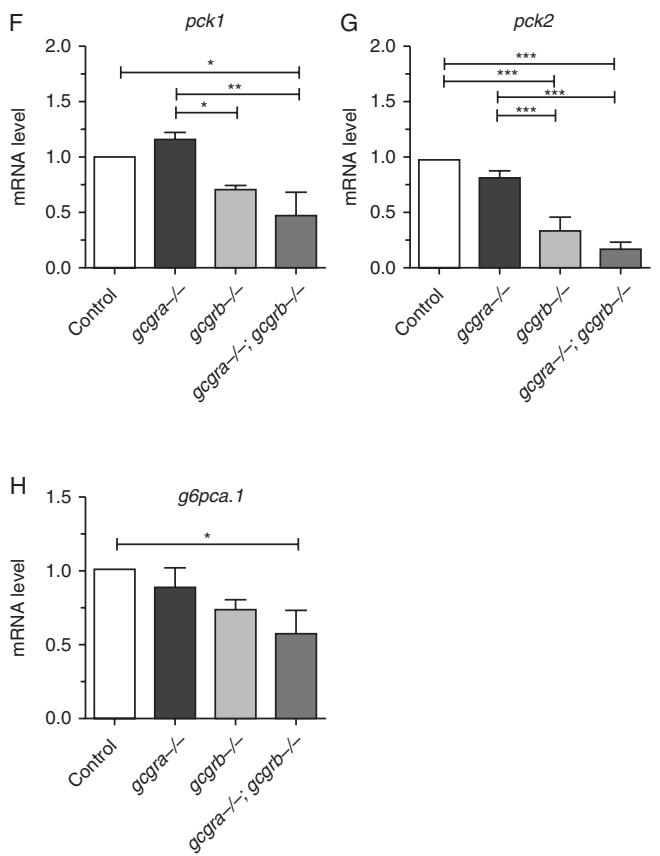

\section{Figure 6}

Defects in glucose metabolism in gcgr-deficient zebrafish at $7 \mathrm{dpf}$. (A, B and C) Real-time PCR analysis of gcga (A), gcgb (B), and insulin (C) mRNA in WT and mutant fish. (D) Total glucagon content in WT and mutant fish. (E) Total free glucose content in WT and mutant fish. $(F, G$ and $H)$ Quantitative RT-PCR analysis of the expression of key gluconeogenic genes pck1 (F), pck2 (G), and g6pca.1 (H). Expression of $\beta$-actin was used as an internal control for all real-time PCR experiments. All the values shown are means \pm s.E.M. from three independent experiments. ${ }^{*} P<0.05, * * P<0.01$, and $* * * P<0.001$ by one-way ANOVA.
We show that $g c g r$-deficient zebrafish display many phenotypes similar to Gcgr-deficient mice, having lower free glucose content and higher glucagon content, similar to the hypoglycemia and hyperglucagonemia seen in mice (Gelling et al. 2003, Vuguin et al. 2006). Importantly, gcgrdeficient zebrafish have a greater $\alpha$-cell mass (Fig. 4) and the increase of $\alpha$-cells is at least partially from $\alpha$-cell replication (Fig. 5). Similar to our results, Ye et al. (2015) reported that knockdown of zebrafish gcga by morpholino resulted in supernumerary $\alpha$-cells partially from proliferation of existing $\alpha$-cells. These data indicate that the compensatory mechanism to glucagon deficiency or interruption of glucagon signaling is conserved in zebrafish. In $g c g r$-deficient mice, increased $\alpha$-cell number is observed as early as day e15 (Vuguin et al. 2006). Similarly, the $\alpha$-cell hyperplasia phenotype is very robust as early as $7 \mathrm{dpf}$ in zebrafish, a stage that is amenable for large-scale genetic and small molecule screens. Therefore, GCGRdeficient zebrafish offer an opportunity to unravel the molecular mechanism of compensatory $\alpha$-cell hyperplasia resulting from glucagon antagonism and to discover other signals that regulate $\alpha$-cell mass.
Supplementary data

This is linked to the online version of the paper at http://dx.doi.org/10.1530/ JOE-15-0284.

\section{Declaration of interest}

The authors declare that there is no conflict of interest that could be perceived as prejudicing the impartiality of the research reported.

\section{Funding}

This work was supported by grants from the American Diabetes Association (1-13-BS-027 to W C), the NIH (DK088686 and GM110311 to W C, DK72473, DK89572, DK89538, DK104211, DK66636, and DK69603 to A C P), by a Merit Review Grant from the Department of Veterans Affairs (to A C P), USDA (AFRI 2014-04938 to W C), and by the Vanderbilt Diabetes Research and Training Center (DK20593) and JDRF (5-2013-111 to A C P and 3-PDF-2014189-A-N to E D D). We utilized the Islet Analysis and Procurement Core of the Vanderbilt Diabetes Research and Training Center (DK02593). Confocal imaging was performed in the VUMC Cell Imaging Shared Resource (supported by NIH grants CA68485, DK20593, DK58404, HD15052, DK59637, and EY08126).

\section{Author contribution statement}

W C is the guarantor of this work and, as such, had full access to all of the data in the study and takes responsibility for the integrity of the data and

Published by Bioscientifica Ltd 
the accuracy of the data analysis. W C, M L, E D D, and A C P designed the study. $M L, E D D, L Z$, and W E N performed the key experiments. W C, M L, $D D, A C P, L Z$, and $W E N$ participated in the planning of the work and the interpretation of the results. $M L$ and $W C$ drafted the manuscript. WC, M L, $D D$, and A C $P$ have participated in revising the paper.

\section{Acknowledgements}

We thank Anastasia Coldren, Chunhua Dai, and members of the Chen lab for constructive discussions, and Amanda Goodrich and Corey Guthrie for excellent fish care.

\section{References}

Abrahamsen N, Lundgren K \& Nishimura E 1995 Regulation of glucagon receptor mRNA in cultured primary rat hepatocytes by glucose and cAMP. Journal of Biological Chemistry 270 15853-15857. (doi:10.1074/ jbc.270.26.15853)

Ali S \& Drucker DJ 2009 Benefits and limitations of reducing glucagon action for the treatment of type 2 diabetes. American Journal of Physiology. Endocrinology and Metabolism 296 E415-E421. (doi:10.1152/ ajpendo.90887.2008)

Argenton F, Zecchin E \& Bortolussi M 1999 Early appearance of pancreatic hormone-expressing cells in the zebrafish embryo. Mechanisms of Development 87 217-221. (doi:10.1016/S09254773(99)00151-3)

Biemar F, Argenton F, Schmidtke R, Epperlein S, Peers B \& Driever W 2001 Pancreas development in zebrafish: early dispersed appearance of endocrine hormone expressing cells and their convergence to form the definitive islet. Developmental Biology 230 189-203. (doi:10.1006/dbio. 2000.0103)

Brubaker PL \& Drucker DJ 2002 Structure-function of the glucagon receptor family of $\mathrm{G}$ protein-coupled receptors: the glucagon, GIP, GLP-1, and GLP-2 receptors. Receptors \& Channels 8 179-188. (doi:10.1080/10606820213687)

Cermak T, Doyle EL, Christian M, Wang L, Zhang Y, Schmidt C, Baller JA, Somia NV, Bogdanove AJ \& Voytas DF 2011 Efficient design and assembly of custom TALEN and other TAL effector-based constructs for DNA targeting. Nucleic Acids Research 39 e82. (doi:10.1093/nar/ gkr218)

Chen M, Gavrilova O, Zhao WQ, Nguyen A, Lorenzo J, Shen L, Nackers L, Pack S, Jou W \& Weinstein LS 2005 Increased glucose tolerance and reduced adiposity in the absence of fasting hypoglycemia in mice with liver-specific Gs $\alpha$ deficiency. Journal of Clinical Investigation $\mathbf{1 1 5}$ 3217-3227. (doi:10.1172/JCI24196)

Chow BK, Moon TW, Hoo RL, Yeung CM, Muller M, Christos PJ \& Mojsov S 2004 Identification and characterization of a glucagon receptor from the goldfish Carassius auratus: implications for the evolution of the ligand specificity of glucagon receptors in vertebrates. Endocrinology 145 3273-3288. (doi:10.1210/en.2003-0597)

Conarello SL, Jiang G, Mu J, Li Z, Woods J, Zycband E, Ronan J, Liu F, Roy RS, Zhu L et al. 2007 Glucagon receptor knockout mice are resistant to diet-induced obesity and streptozotocin-mediated $\beta$ cell loss and hyperglycaemia. Diabetologia 50 142-150. (doi:10.1007/s00125-0060481-3)

Cruz SA, Tseng YC, Kaiya H \& Hwang PP 2010 Ghrelin affects carbohydrate-glycogen metabolism via insulin inhibition and glucagon stimulation in the zebrafish (Danio rerio) brain. Comparative Biochemistry and Physiology. Part A, Molecular \& Integrative Physiology 156 190-200. (doi:10.1016/j.cbpa.2010.01.019)

Field HA, Dong PD, Beis D \& Stainier DY 2003 Formation of the digestive system in zebrafish. II. Pancreas morphogenesis. Developmental Biology 261 197-208. (doi:10.1016/S0012-1606(03)00308-7)
Gelling RW, Du XQ, Dichmann DS, Romer J, Huang H, Cui L, Obici S, Tang B, Holst JJ, Fledelius C et al. 2003 Lower blood glucose, hyperglucagonemia, and pancreatic $\alpha$ cell hyperplasia in glucagon receptor knockout mice. PNAS 100 1438-1443. (doi:10.1073/pnas. 0237106100)

Hansen LH, Abrahamsen N \& Nishimura E 1995 Glucagon receptor mRNA distribution in rat tissues. Peptides 16 1163-1166. (doi:10.1016/01969781(95)00078-X)

Hesselson D, Anderson RM \& Stainier DY 2011 Suppression of Ptf1a activity induces acinar-to-endocrine conversion. Current Biology 21 712-717. (doi:10.1016/j.cub.2011.03.041)

Jiang G \& Zhang BB 2003 Glucagon and regulation of glucose metabolism. American Journal of Physiology. Endocrinology and Metabolism 284 E671-E678. (doi:10.1152/ajpendo.00492.2002)

Kedees MH, Grigoryan M, Guz Y \& Teitelman G 2009 Differential expression of glucagon and glucagon-like peptide 1 receptors in mouse pancreatic $\alpha$ and $\beta$ cells in two models of $\alpha$ cell hyperplasia. Molecular and Cellular Endocrinology 311 69-76. (doi:10.1016/j.mce.2009.07.024)

Kimmel CB, Ballard WW, Kimmel SR, Ullmann B \& Schilling TF 1995 Stages of embryonic development of the zebrafish. Developmental Dynamics 203 253-310. (doi:10.1002/aja.1002030302)

Lee Y, Wang MY, Du XQ, Charron MJ \& Unger RH 2011 Glucagon receptor knockout prevents insulin-deficient type 1 diabetes in mice. Diabetes 60 391-397. (doi:10.2337/db10-0426)

Li M, Maddison LA, Crees Z \& Chen W 2013 Targeted overexpression of CKI-insensitive cyclin-dependent kinase 4 increases functional $\beta$-cell number through enhanced self-replication in zebrafish. Zebrafish $\mathbf{1 0}$ 170-176. (doi:10.1089/zeb.2012.0816)

Li M, Maddison LA, Page-McCaw P \& Chen W 2014 Overnutrition induces $\beta$-cell differentiation through prolonged activation of $\beta$-cells in zebrafish larvae. American Journal of Physiology. Endocrinology and Metabolism 306 E799-E807. (doi:10.1152/ajpendo.00686.2013)

Lieschke GJ \& Currie PD 2007 Animal models of human disease: zebrafish swim into view. Nature Reviews. Genetics 8 353-367. (doi:10.1038/ $\operatorname{nrg} 2091)$

Livak KJ \& Schmittgen TD 2001 Analysis of relative gene expression data using real-time quantitative PCR and the $2(-$ Delta Delta $C(\mathrm{~T}))$ method. Methods 25 402-408. (doi:10.1006/meth.2001.1262)

Longuet C, Robledo AM, Dean ED, Dai C, Ali S, McGuinness I, de Chavez V, Vuguin PM, Charron MJ, Powers AC et al. 2013 Liver-specific disruption of the murine glucagon receptor produces $\alpha$-cell hyperplasia: evidence for a circulating $\alpha$-cell growth factor. Diabetes 62 1196-1205. (doi:10.2337/db11-1605)

Lund A, Bagger JI, Christensen M, Knop FK \& Vilsboll T 2014 Glucagon and type 2 diabetes: the return of the $\alpha$ cell. Current Diabetes Reports 14555. (doi:10.1007/s11892-014-0555-4)

Maddison LA \& Chen W 2012 Nutrient excess stimulates $\beta$-cell neogenesis in zebrafish. Diabetes 61 2517-2524. (doi:10.2337/db11-1841)

Mayo KE, Miller LJ, Bataille D, Dalle S, Goke B, Thorens B \& Drucker DJ 2003 International Union of Pharmacology. XXXV. The glucagon receptor family. Pharmacological Reviews 55 167-194. (doi:10.1124/pr. 55.1.6)

Ni TT, Lu J, Zhu M, Maddison LA, Boyd KL, Huskey L, Ju B, Hesselson D, Zhong TP, Page-McCaw PS et al. 2012 Conditional control of gene function by an invertible gene trap in zebrafish. PNAS $\mathbf{1 0 9}$ 15389-15394. (doi:10.1073/pnas.1206131109)

Venkatesh B 2003 Evolution and diversity of fish genomes. Current Opinion in Genetics \& Development 13 588-592. (doi:10.1016/j.gde.2003.09.001)

Vuguin PM, Kedees MH, Cui L, Guz Y, Gelling RW, Nejathaim M, Charron MJ \& Teitelman G 2006 Ablation of the glucagon receptor gene increases fetal lethality and produces alterations in islet development and maturation. Endocrinology 147 3995-4006. (doi:10.1210/en.20051410)

Ye L, Robertson MA, Hesselson D, Stainier DY \& Anderson RM 2015 Glucagon is essential for $\alpha$ cell transdifferentiation and $\beta$ cell neogenesis. Development 142 1407-1417. (doi:10.1242/dev.117911) 
Yu R, Dhall D, Nissen NN, Zhou C \& Ren SG 2011 Pancreatic neuroendocrine tumors in glucagon receptor-deficient mice. PLoS ONE 6 e23397. (doi:10.1371/journal.pone.0023397)

Zecchin E, Filippi A, Biemar F, Tiso N, Pauls S, Ellertsdottir E, Gnugge L, Bortolussi M, Driever W \& Argenton F 2007 Distinct delta and jagged genes control sequential segregation of pancreatic cell types from precursor pools in zebrafish. Developmental Biology 301 192-204. (doi:10.1016/j.ydbio.2006.09.041)

Zhou C, Dhall D, Nissen NN, Chen CR \& Yu R 2009 Homozygous P86S mutation of the human glucagon receptor is associated with hyperglucagonemia, $\alpha$ cell hyperplasia, and islet cell tumor. Pancreas 38 941-946. (doi:10.1097/MPA.0b013e3181b2bb03)

Received in final form 21 August 2015

Accepted 1 September 2015
Published by Bioscientifica Ltd. 